\title{
Continuing education in therapeutics: aids to discussions on prescribing
}

\author{
ANDREW HERXHEIMER, ROBERT TWYCROSS
}

\section{British Medical fournal, 1976, 1, 1198-1200}

Specialists in pharmacotherapeutics have recently been taken to task for being engrossed in matters of dubious clinical relevance and for not showing more concern with educating doctors in how to use drugs properly.' Consequently, many doctors still depend largely on drug companies for information about drugs, and lack the opportunity to discuss therapeutic choices and problems with their colleagues. At postgraduate medical centres diagnosis and investigation dominate the programmes, ${ }^{2}$ and even when therapeutic problems are aired they tend to be presented didactically by specialists who may be unaware of the needs of general practice. Moreover, such an approach violates the principle that learners should be active participants in the educational process. ${ }^{3}$

We describe two methods which we have found useful at postgraduate meetings in helping doctors to discuss their prescribing with each other. Because both are rooted in the individual doctor's experience everyone may take part. The first method makes use of precirculated questions, the second of partially completed decision flow-charts.

\section{Precirculated questions}

The purpose of the questions is to elicit what the participants prescribe in certain conditions. Both specific and open-ended questions are used. The questions are sent out one to two weeks before the meeting and, ideally, replies should be returned to the tutor before the meeting to help him decide how to structure the discussion.

Examples of specific questions are:

(1) (a) What antibacterial drugs do you use for patients with chronic bronchitis, and how do you choose between them for a particular patient? (b) What do you tell the patient about the preparation prescribed? (c) In what situations do you decide not to use an antibacterial at all ?

(2) (a) What preparations do you use to treat urinary infection and how do you choose between them for a particular patient? (b) For how long do you prescribe these drugs, and how do you decide when to stop?

(3) In patients with migraine, $(a)$ what preparations do you prescribe for use in the acute attack? (b) what do you prescribe for interval treatment, to prevent or reduce the frequency of attacks ? At what intervals do you ask to see these patients?

Our favourite open-ended question is:

(4) What preparations have you started to use for the first time during the last six/twelve months ? For each one you list, state: $(a)$ the purpose for which you use it, $(b)$ the treatment you previously used, and $(c)$ what made you change.

This is not a question about new drugs as such but one about preparations not previously used by the prescriber.

Before a session dealing with unwanted effects a converse question may be more appropriate.

(5) During the last twelve months what preparations $(a)$ have you stopped using altogether, and why ? $(b)$ are you now using less freely than before, and why ? What are you using in the place of each of these preparations ?

It is also useful to ask:

(6) Have you met any serious or troublesome adverse reactions to drugs in the last year? Please describe them briefly.

Lastly, it may be worth asking about non-drug treatments.

(7) Have you in the past year begun to use, or to refer patients for, any

Department of Pharmacology and Therapeutics, London Hospital Medical College, London E1 2AD

ANDREW HERXHEIMER, MB, MRCP, senior lecture

ROBERT TWYCROSS, MB, MRCP, honorary lecturer non-drug treatments that were new to vou ? Please say what they are, for what conditions you have used them, and what you previously used for these conditions.

\section{Partially completed decision flow-chart}

Decision flow-charts, also called algorithms or decision trees, have long been used in industrial management and other disciplines, but are relatively new to medicine. ${ }^{4}$ They aim to clarify the essential issues on which decisions must be made, their order, and their likely consequences. Examples are given which describe decision sequences for managing pain in rheumatoid arthritis (fig 1) and advanced malignant disease (fig 2 ). The tutor begins by posing a specific question-for instance, "A patient with terminal cancer complains of mild pain: what would you do ?" The participants then say what they would prescribe and a general discussion of the various possibilities ensues. All the other decision points are discussed in sequence. About an hour and a half is needed for a fairly full discussion of the examples in figs 1 or 2 . If less time is available the discussion may be limited to selected aspects. The value of the seminar may be enhanced by distributing notes at the end which have been prepared by the tutor.

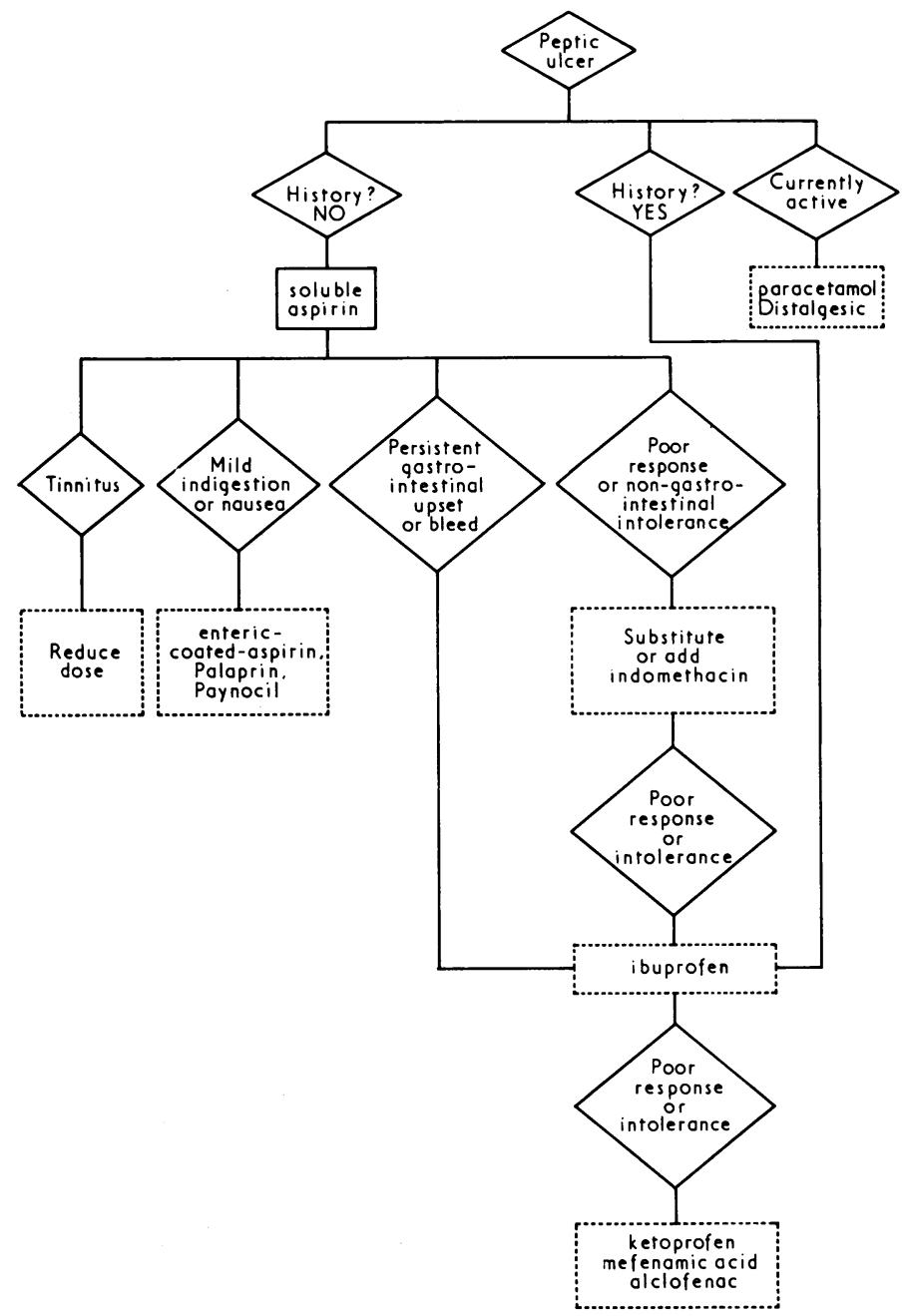

FIG 1-Choice of Anti-inflammatory drugs in rheumatoid arthritis (adapted from Drug Therapeutics Bulletin ${ }^{5}$ ). Diamonds indicate findings, rectangles decisions. The rectangles with a broken outline are those which were left blank when the flow-chart was first presented to the group. 


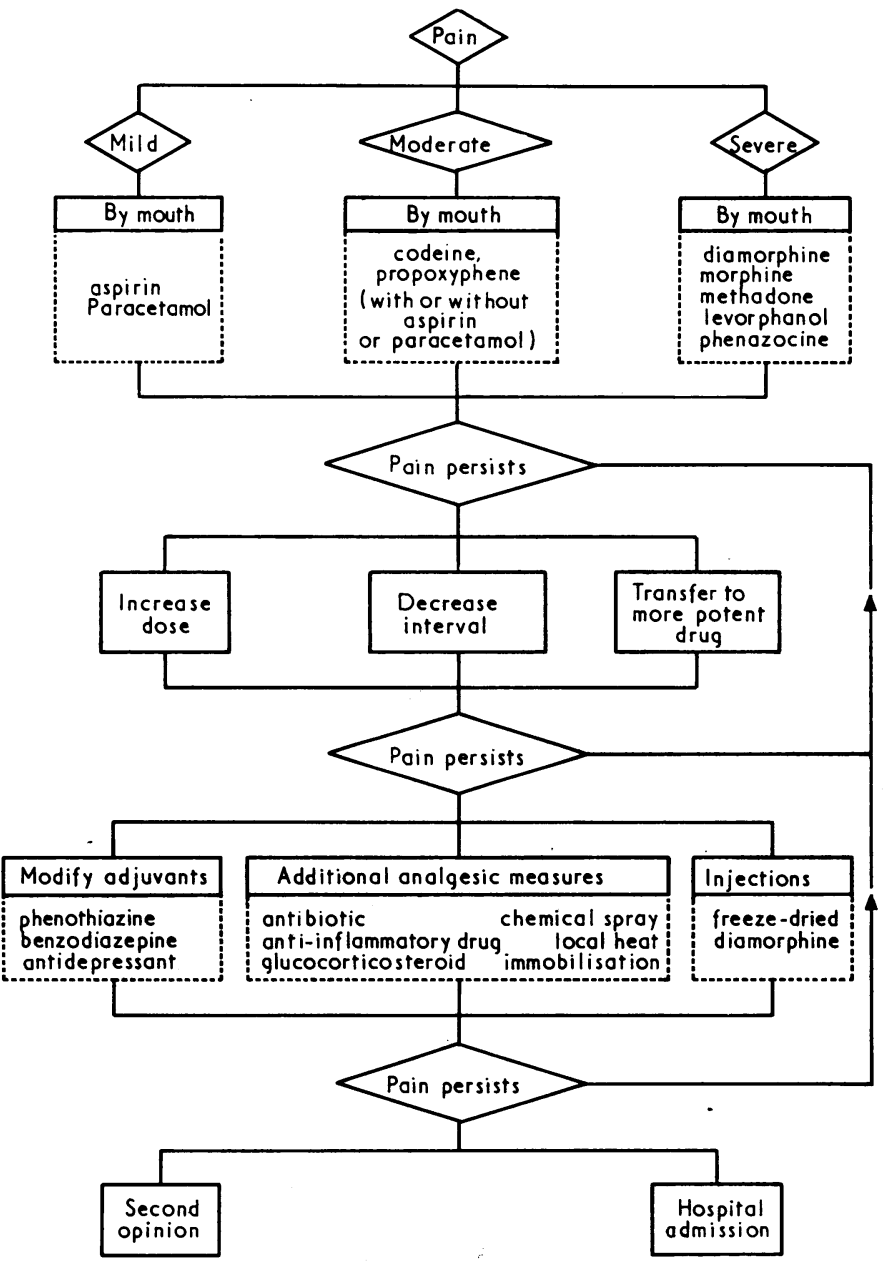

FIG 2-Management of pain in malignant disease. Conventions as for fig 1.

\section{Discussion}

The precirculated questions and decision flow charts simplify the clinical problems in order to focus attention on some of the decisions to be discussed. In clinical practice other factors will, of course, have to be considered and may influence the choice of treatment. Moreover, intentional ambiguities in the questions or gaps in the decision sequence enhance, rather than detract from, the value of the discussion. For example, question $1(a)$ does not distinguish between healing an acute exacerbation of chronic bronchitis and prophylactic antibacterial treatment. The ambiguity enables the tutor to discover how clearly the participants distinguish between distinct though related problems.

The decision flow-chart, likewise, is not comprehensive. For example, although pain is often classified as mild, moderate, or severe, most doctors use a four-point "analgesic ladder" and would use a drug such as dipipanone, papaveretum, or dextromoramide before prescribing morphine or diamorphine. It may be necessary to spend some time considering the nature of underlying pathological states. Sometimes it is important to relate drug treatment to non-drug treatment.

An important prerequisite for using either method is that the participants should have had clinical experience of the problem to be discussed. We have used the methods with general practitioners, young hospital doctors, and groups containing both. Difficulties may arise if the experience of the participants is too specialised and diverse. The methods may be used in groups of any size up to about 50 people; in larger groups it is not possible for everybody to take part in the discussion. We originally used these methods at meetings where the participants did not know each other, partly with the aim of including everyone in the discussion from the start. But the methods are also useful in groups that meet repeatedly-for example, a fortnightly seminar in therapeutics for general practitioners. The more detailed discussion, with more preparatory work between meetings, facilitates the development of coherent therapeutic policies by the group.

\section{References}

1 British Medical fournal, 1976, 1, 59.

2 Report of the Working Party on the Continuing Education of Doctors in Medicinal Therapeutics. Medico-Pharmaceutical Forum, London, 1975. 3 British Medical fournal, 1976, 1, 116.

4 Feinstein, A R, Yale fournal of Biology and Medicine, 1974, 47, 5.

5 Drug and Therapeutics Bulletin, 1975, 13, 57.
$A$ boy had a triple antigen injection in infancy; at school entry (age 9) he had a severe reaction with high fever to the booster tetanus toxoid. There was no local reaction. He is now adult. If he is injured would it be advisable for him to receive further tetanus toxoid injections?

A course of at least three doses of triple vaccine during infancy, followed by a booster dose of tetanus vaccine when aged 9 , probably induced long-term but not necessarily life-long immunity to tetanus. Since he is now adult and his serum tetanus antitoxin has possibly waned to a subprotective level, a single booster dose of tetanus toxoid is advisable to establish life-long immunity. High fever after a booster dose of tetanus toxoid when 9 is not a contraindication to another booster dose many years later.

What evidence is there that chronic anxiety and stress help cause coronary thrombosis?

There is no evidence that chronic anxiety and stress contribute to chronic atheroma and myocardial infarction, although much work (mainly epidemiological) has been done into finding out whether such an association exists. It has been alleged that the adrenaline released during mental stress leads to consequences such as tachycardia, fatty acid release, and platelet aggregation, which may be desirable during physical exertion but not when no exercise is taken. Coronary heart disease results from multiple rather than a single cause. Recently, interest has centred more on the protective effects of regular physical exercise than on the deleterious effects of anxiety. The incidence of coronary heart disease has been examined among individuals with a type " $A$ " personality (ambitious, aggressive, and tense) and found to be higher than in type " $B$ " individuals (more easy-going and less successful), but this work should perhaps not be taken too seriously and hard work, ambition, and drive should certainly not be suppressed in our efforts to prevent ischaemic heart disease.

What is known about treating malignant tumours by local hyperthermia?

Heating to $45^{\circ} \mathrm{C}$ readily kills cells in culture, especially if they are hypoxic. There is some evidence that cancer cells are more susceptible to destruction by heat than normal cells. ${ }^{1}$ Local hyperthermia of limbs to about $41-42^{\circ} \mathrm{C}$ for short periods is safe and sometimes results in definite regression of cancers, ${ }^{2}$ especially when applied with chemotherapeutic agents. Local hyperthermia, however, also tends to cause considerable toxic effects and heating to an inadequately high temperature may favour cancer growth rather than retard it. ${ }^{1}$ There are extensive reports on the possible role of heat in cancer treatment and research is actively in progress. In the meantime it would be unwise for anyone unfamiliar with the subject to experiment with hyperthermia in treating any patient with cancer. ${ }^{3}$

1 Dickson, J A, Cancer Chemotherapy Reports part 1, 1974, 58, 294.

2 Cavaliere, R, Ciocatto, E C, and Giovanella, B C, Cancer, 1967, 20, 1351 3 Holman, R A, Lancet, 1975, 1, 1027. 